\title{
TOPS Imaging With TerraSAR-X: Mode Design and Performance Analysis
}

\author{
Adriano Meta, Member, IEEE, Josef Mittermayer, Member, IEEE, Pau Prats, Member, IEEE, \\ Rolf Scheiber, and Ulrich Steinbrecher
}

\begin{abstract}
This paper reports about the performed investigations for the implementation of the wide-swath TOPS (Terrain Observation by Progressive Scan) imaging mode with TerraSAR-X (TSX). The TOPS mode overcomes the limitations imposed by the ScanSAR mode by steering the antenna along track during the acquisition of a burst. In this way, all targets are illuminated with the complete azimuth antenna pattern, and, thus, scalloping is circumvented, and an azimuth dependence of signalto-noise ratio and distributed target ambiguity ratio (DTAR) is avoided. However, the use of electronically steered antennas leads to a quantization of the steering law and a nonideal pattern for squinted angles (grating lobes and main lobe reduction). The former provokes spurious peaks, while the latter introduces slight scalloping and DTAR deterioration. These effects are analyzed and quantified for TSX, and a TOPS system design approach is presented. Next, the requirements concerning interferometry are investigated. Finally, several results are shown with the TSX data, including a comparison between the TOPS and the ScanSAR modes and the reporting of the first TOPS interferometric results.
\end{abstract}

Index Terms-ScanSAR, synthetic aperture radar (SAR), terrain observation by progressive scan (TOPS), wide-swath SAR.

\section{INTRODUCTION}

$\mathbf{T}$ HE Terrain Observation by Progressive Scan (TOPS) mode [1] is a new and promising mode of wide-swath synthetic aperture radar (SAR) operation for future SAR satellite missions. Up to now, ScanSAR is the established mode in SAR for wide-swath imaging. However, the mode has several disadvantages caused by the focusing of targets from different portions of the azimuth antenna pattern. These antenna pattern slices are moving within the $3-\mathrm{dB}$ azimuth beamwidth depending on the target position in azimuth [10]. The drawbacks in ScanSAR are a variation of signal-to-noise ratio (SNR) and azimuth ambiguity ratio along azimuth within the processed burst images, as well as scalloping, which is an amplitude modulation along the assembled bursts in azimuth [7], [10], [11].

TOPS is an alternative acquisition mode to achieve the same swath coverage as in ScanSAR but drastically reducing its drawbacks. The technique is employing a rotation of the

Manuscript received February 4, 2009; revised April 24, 2009. First published October 6, 2009; current version published January 20, 2010. This work was supported by the European Space Agency Contract C20679/07/NL/BC.

A. Meta was with the German Aerospace Center, 82230 Wessling, Germany. He is now with MetaSensing, 2201 AZ Noordwijk, The Netherlands.

J. Mittermayer, P. Prats, R. Scheiber, and U. Steinbrecher are with the Microwaves and Radar Institute, German Aerospace Center, 82230 Wessling, Germany (e-mail: josef.mittermayer@dlr.de).

Color versions of one or more of the figures in this paper are available online at http://ieeexplore.ieee.org.

Digital Object Identifier 10.1109/TGRS.2009.2026743

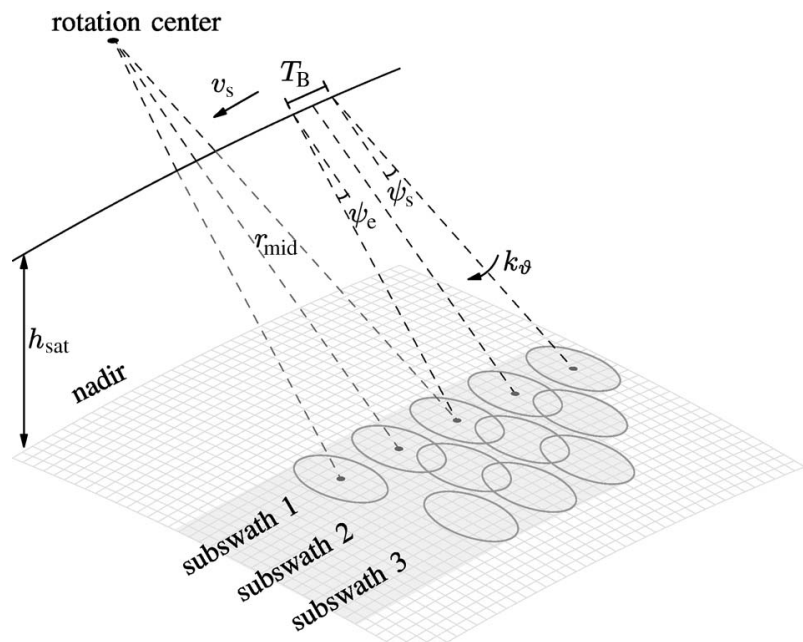

Fig. 1. Sketch of the acquisition geometry in the TOPS imaging mode. $T_{B}$ is the burst duration, $r_{\text {mid }}$ is the midrange slant range distance in a given subswath, $v_{s}$ is the satellite velocity, $h_{\text {sat }}$ is the satellite altitude, $k_{\vartheta}$ is the steering angle rate, and $\phi_{s}$ and $\phi_{e}$ are the starting and the end steering angles within the burst, respectively.

antenna in the azimuth direction, as is shown in Fig. 1. Just like in ScanSAR, from a small burst of raw data, a much longer burst image is focused, and, thus, several subswaths can be acquired by subswath switching from burst to burst. The increased swath coverage is achieved by the tradeoff with a reduced azimuth resolution. However, in TOPS, the resolution reduction is obtained by a virtual shrinking of the antenna footprint, as seen by an on-ground target, rather than slicing the antenna pattern, as it happens for ScanSAR.

TOPS has been selected as the baseline mode for European Space Agency (ESA)'s Sentinel-1 SAR system [2]. In the frame of an ESA contract [3], a complete performance analysis and a demonstration of the TOPS mode with TerraSAR-X (TSX) [17], [18] were carried out. This was possible since TSX is a versatile space SAR platform. Its antenna steering capability, together with the flexible instrument operation and calibration section concept [5], [19], offers the opportunity to implement different acquisition modes, as is demonstrated by the TOPS mode acquisitions presented in this paper. The design aspects and the representative TSX demonstration results are discussed.

Section II starts with a brief summary of the TOPS acquisition mode. The inverse TOPS mode is introduced to support the following discussion on the impact of electronic azimuth beam steering and scalloping. For electronically steered antennas, the boundaries to the angular quantization are derived. Based on these fundamental investigations, the design methodology for 
a TOPS mode acquisition is developed, i.e., in which sequence the acquisition parameters need to be calculated. An acquisition parameter set is then derived for TSX. In Section III, the special requirements on the interferometric TOPS performance are presented with respect to burst alignment and coregistration accuracy. Finally, in Section IV, the experimental results of the TSX TOPS data acquisition are presented.

\section{TOPS SYSTEM DESIGN}

\section{A. TOPS Principle Overview}

In TOPS, the prolongation of the focused burst image with respect to the raw data burst is achieved by the antenna steering in azimuth (see Fig. 1). This results in a reduced azimuth resolution due to the reduction in the dwell time of the antenna footprint on every target within the swath. The Doppler bandwidth acquired for each target in the swath is no longer directly related only to the burst time $T_{B}$ but also to the antenna steering angle rate $k_{\vartheta}$. In ScanSAR, the dwell time, i.e., the azimuth integration time for a point target, is identical to the burst time. In TOPS, the burst time is longer than the dwell time.

Assuming a sinc azimuth pattern, the resulting TOPS antenna pattern as seen by a point target is [1]

$$
\begin{aligned}
G_{T}(\vartheta(\tau)) & =G_{0} \operatorname{sinc}^{2}\left(\frac{L}{\lambda} \cdot\left(\frac{v \tau}{R_{0}}+\vartheta(\tau)\right)\right) \\
& \approx G_{0} \operatorname{sinc}^{2}\left(\frac{L v \tau}{\lambda R_{0}} \cdot\left(1+\frac{R_{0} k_{\vartheta}}{v}\right)\right)
\end{aligned}
$$

where $\vartheta(\tau)$ is the antenna rotation angle as a function of slow time $\tau, v$ is the ground velocity, $\lambda$ is the wavelength, $R_{0}$ is the range of the closest approach, and $L$ is the physical azimuth antenna length.

The resulting TOPS azimuth resolution is reduced with respect to the stripmap resolution by a factor that is equal to

$$
\alpha=1+\frac{R_{0} k_{\vartheta}}{v}
$$

leading to

$$
\rho_{\mathrm{az}}^{\mathrm{TOPS}}=\rho_{\mathrm{az}}^{\mathrm{STRIP}} \cdot \alpha=\frac{\lambda}{2 \vartheta_{0}} \cdot\left(1+\frac{R_{0} k_{\vartheta}}{v}\right)
$$

where $\vartheta_{0}$ is the antenna azimuth beamwidth exploited for focusing the SAR data. The choice of $\vartheta_{0}$ is a tradeoff between resolution (requiring a large aperture angle) and ambiguity suppression and SNR (requiring a small aperture angle). Different values of $\vartheta_{0}$ can be chosen for the different subswaths. Once the value of $\vartheta_{0}^{n}$ is fixed (the apex $n$ refers to the $n$th subswath), the steering angle parameters can be derived by inverting (3) [1]

$$
k_{\vartheta}^{n}=\left(\frac{2 \rho_{\mathrm{az}} \vartheta_{0}^{n}}{\lambda}-1\right) \frac{v}{R_{0}^{n}} .
$$

After the steering angle rates have been calculated, the global TOPS timeline can be derived, imposing a cycle time $T_{R}$ that is sufficiently small to assure along-track gap-free subswath coverage. This constraint results in a set of $n$ linear equations [1]

$$
\left(k_{\vartheta}^{n} T_{B}^{n}-\vartheta_{0}^{n}\right) R_{0}^{n}+v^{n} T_{B}^{n}=v^{n} T_{R}
$$

where

$$
T_{R}=\sum_{n} T_{B}^{n}+T_{G}^{n}
$$

and $T_{G}^{n}$ is a time margin to assure sufficient overlapping of consecutive focused burst images of one subswath. In case the overlapping is too small, the steering rate needs to be increased, and, thus, the overlap is traded against the azimuth resolution.

\section{B. Inverse TOPS}

The basic principle of TOPS is the reduction in the dwell time of the azimuth antenna footprint on every target within the swath obtained by steering the antenna in a direction that is opposite to the direction for spotlight. The same reduction in the dwell time can be obtained by steering the antenna in the same direction as in spotlight with a rotation center between the platform and the scene. Wide-swath images can thus be acquired also in an inverse TOPS mode [15]. Both TOPS and inverse TOPS have the same burst length for a given resolution [14]. However, as in inverse TOPS, the satellite velocity is working against the antenna steering; a larger steering angle range and higher steering rates are required. This causes, generally, a performance loss compared to the TOPS mode when using electronically steered antennas. Scalloping performance plots are presented for TOPS and inverse TOPS in Section IV-B.

Concerning the analytical analysis of inverse TOPS, the value of the steering rate $k_{i \vartheta}$ relative to a TOPS $k_{\vartheta}$ can be found by imposing

$$
\left|1+\frac{k_{\vartheta} R_{0}}{v}\right|=\left|1-\frac{k_{i \vartheta} R_{0}}{v}\right|
$$

which results in

$$
k_{i \vartheta}=\frac{2 v}{R_{0}}+k_{\vartheta}
$$

i.e., the steering rate is higher than that in TOPS. Finally, the same set of linear equations shown in (5) must be solved in order to obtain the timeline.

It is worthwhile to mention that, for one target, different parts of the azimuth spectrum will be acquired in TOPS as well as inverse TOPS modes, depending on the target azimuth position.

\section{Impact of Electronic Azimuth Beam Steering}

TOPS was developed to overcome the ScanSAR performance variation along azimuth, which is a consequence of integrating different azimuth pattern slices depending on the azimuth target position. Thus, ScanSAR images suffer an azimuth variation of SNR and azimuth ambiguities. However, by looking to a ScanSAR image with a low SNR, scalloping is the strongest observable effect.

A realization of the TOPS mode with a mechanical beam steering in the azimuth overcomes the ScanSAR performance variation along azimuth in wide-swath images. Gain variations due to different slant range distances can be neglected. A practical implementation challenge is the platform stability while switching from forward to backward illumination during subswath transition. A combination of TOPS and inverse TOPS could be very helpful in solving this problem. 


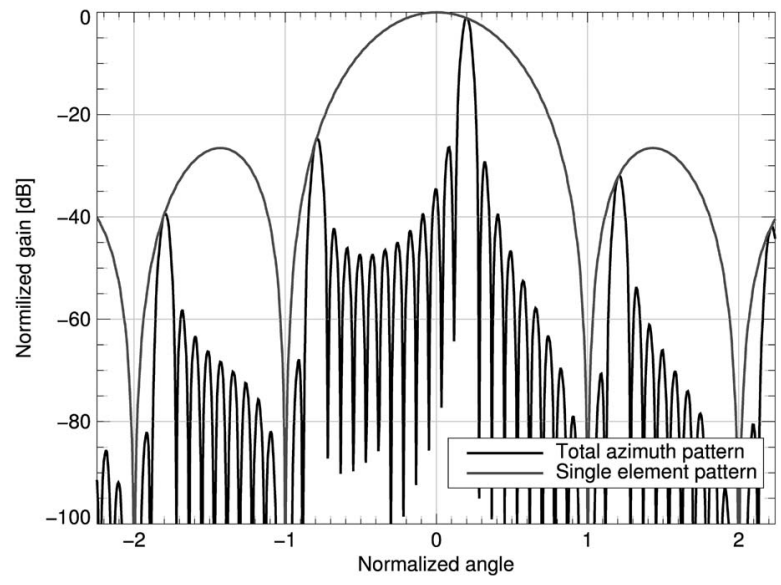

Fig. 2. Element and total antenna pattern for a steering angle of 0.2 (normalized to the first null position of the element pattern).

An advantage of satellite SAR systems with electronic beam steering is the absence of platform stability problems during antenna steering. However, there is a small azimuth variation in SNR and azimuth ambiguity, as well as slight scalloping [12]. This is demonstrated in Fig. 2, which presents, for TSX, the element and the total antenna pattern in azimuth. The total azimuth pattern results from weighting the array pattern by the single element pattern. When the pointing angle is steered away from boresight, grating lobes arise. With increasing steering angle, the grating lobe gain increases, and the main lobe gain decreases. This is the reason for the small scalloping, as well as the small azimuth variation of SNR and azimuth ambiguities, and needs to be considered in the TOPS system design.

An interesting distinction of ScanSAR and TOPS scalloping in electronic steering is that, in TOPS, the scalloping effect is induced by the reduced gain of the main lobe dominated by the element pattern. In ScanSAR, scalloping results from the gain variation within the nonsteered azimuth pattern which is dominated by the array pattern.

Residual TOPS scalloping can be removed with conventional ScanSAR techniques, i.e., Doppler-centroid-based azimuth pattern correction. Furthermore, since TOPS bursts are much longer than those in ScanSAR, scalloping removal is less sensitive to inaccuracies in the Doppler centroid knowledge. The results of scalloping image analysis with TSX are presented in Section IV-B.

Another factor in electronically steered TOPS which needs to be considered is the quantization of the antenna steering angles, which is discussed in the next section.

\section{Steering Angle Quantization}

In orbital SAR systems, electronically steered antennas use a limited number of azimuth steering angles. Thus, the steering angle versus the azimuth time shows typically a staircase behavior with a steering angle step size $\Delta \vartheta$.

Such quantization causes an amplitude modulation of every target in the raw data. The resulting antenna gain as seen by a point target on the ground is plotted in Fig. 3. Such an amplitude modulation distorts the target impulse response function (IRF) by introducing spurious peaks. In TOPS, the amplitude modulation in the raw data of a point target is equal

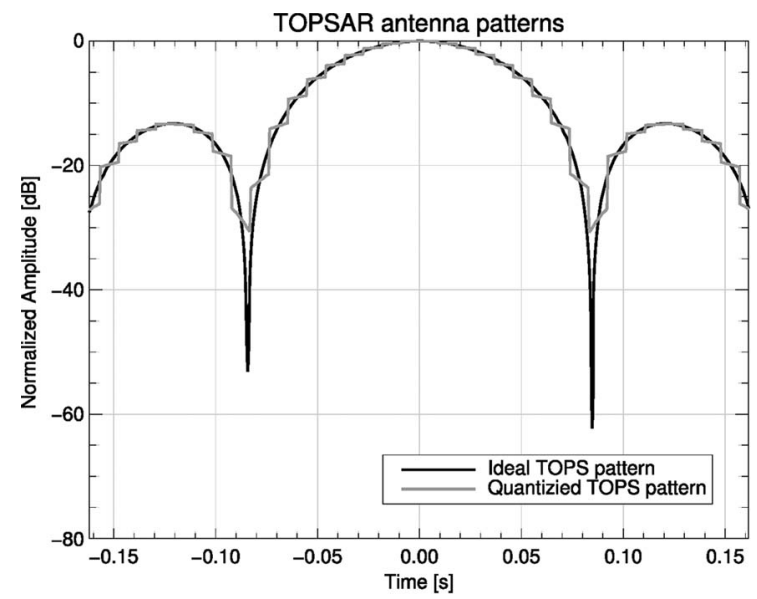

Fig. 3. Effects of steering angle quantization on the TOPS antenna gain as seen by a point target.

to the difference between the uniform and the quantized antenna gain. An analytical solution of the retrieved maximum spurious level is derived in the Appendix.

For the example in Fig. 3, the ideal TOPS IRF, the IRF for quantized steering, and the analytical curve for the amplitude error given in (14) are plotted in Fig. 4(a), proving that the analytical solution is in accordance with the quantized antenna pattern. The quantization step size is $0.03^{\circ}$, and the azimuth resolution is $16 \mathrm{~m}$. No windowing for sidelobe suppression was applied.

The level of the first (and maximum) spurious peak can be derived from (16) in the Appendix. The maximum of the spurious peak does not depend on range and is plotted in Fig. 4(b) for the example in Fig. 3. The plot shows that, for values of the quantization angle smaller than $0.05^{\circ}$, the resulting response distortion induced by the amplitude modulation is negligible.

When using windowing for sidelobe suppression, the distortion peaks are decreased because the maximum error at the end of the processed point target bandwidth is reduced. An improvement of about $5 \mathrm{~dB}$ in the spurious peak level is obtained with a Hamming window, spanning the 3-dB integration angle for the example presented in this section.

\section{E. System Design Methodology}

Fig. 5 shows the block diagram of the procedure to compute the TOPS acquisition parameters. It is slightly different than the one presented in [16], where the performance parameters are calculated for several sets of acquisition parameters. Based on a predefined fitness function, the best acquisition parameter set is then selected. However, in this paper, the calculation of the acquisition parameters considers as input the established performance requirements, which already include tradeoffs between the different performance parameters.

For a given distributed target ambiguity ratio (DTAR) and $\mathrm{SNR}$, the processed beamwidth is selected, which, together with the azimuth resolution, imposes the required steering rates for every subswath. Afterward, the TOPS timeline is computed by solving the system of equations given by (5), which gives the burst lengths for the different subswaths. During the flow, two checks are made. The first one is placed after the derivation 


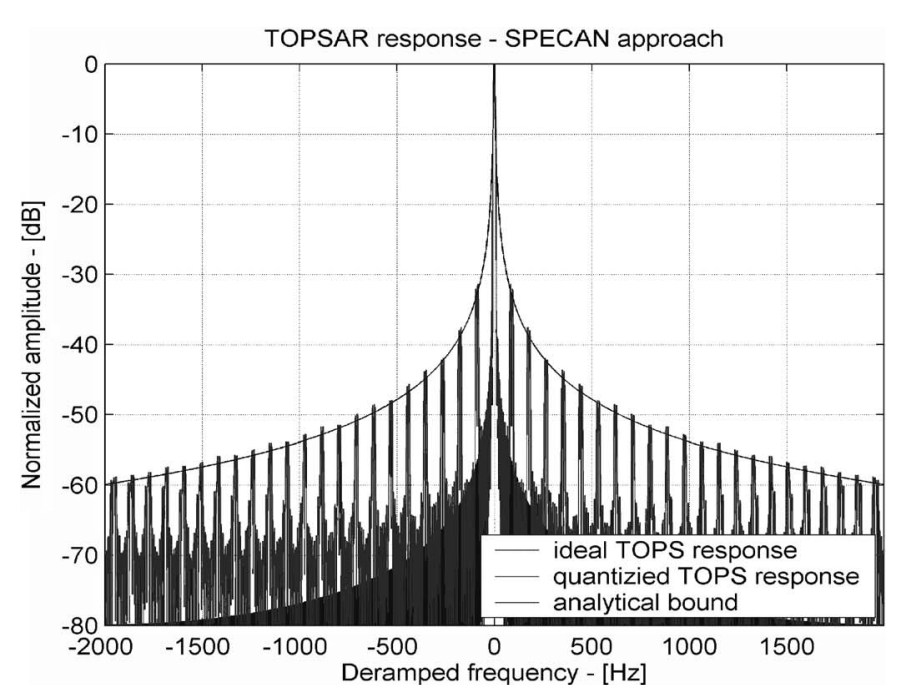

(a)

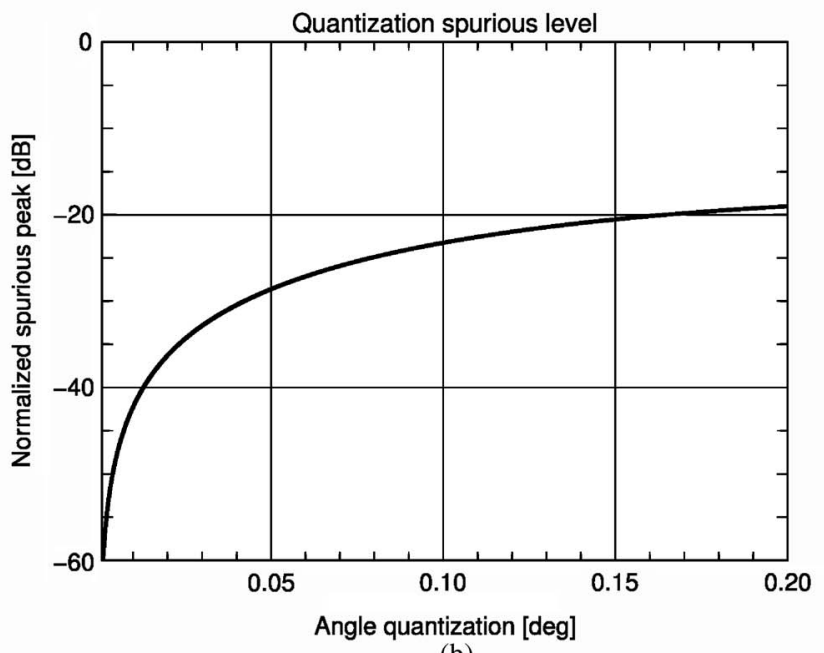

(b)

Fig. 4. (a) TOPS response (IRF) for the ideal uniform antenna and the quantized antenna gain case. In the picture, the error analytical bound is also plotted. A TSX case is simulated with quantization steps of $0.03^{\circ}$ and a resolution of $16 \mathrm{~m}$. (b) Maximum spurious peak level versus angle quantization. The curve is independent on the range distance and the azimuth resolution.

of the maximum required steering angle (given by the burst time and the steering rate for that burst) and ensures that the maximum steering angle imposed by the SAR antenna system (which can be steered up to a certain degree by design constraints like grating lobes) is not exceeded. The second one is placed after the recalculation of the SNR and the DTAR by taking into account the effects of the electronically steered antenna (gain reduction in the main lobe and gain increase in the grating lobes). If, in any of these two cases, the requirements are not met, the processed beamwidth has to be changed in order to reduce the maximum steering angle (by increasing the processed beamwidth) or to improve the SNR and the DTAR (by decreasing the processed beamwidth).

\section{F. System Design Example With TSX}

Using the theoretical analysis and the design tools discussed in the previous sections, the acquisition parameter calculation and the performance estimation are done for the TSX TOPS acquisition mode. The main TSX parameters [5] are listed in

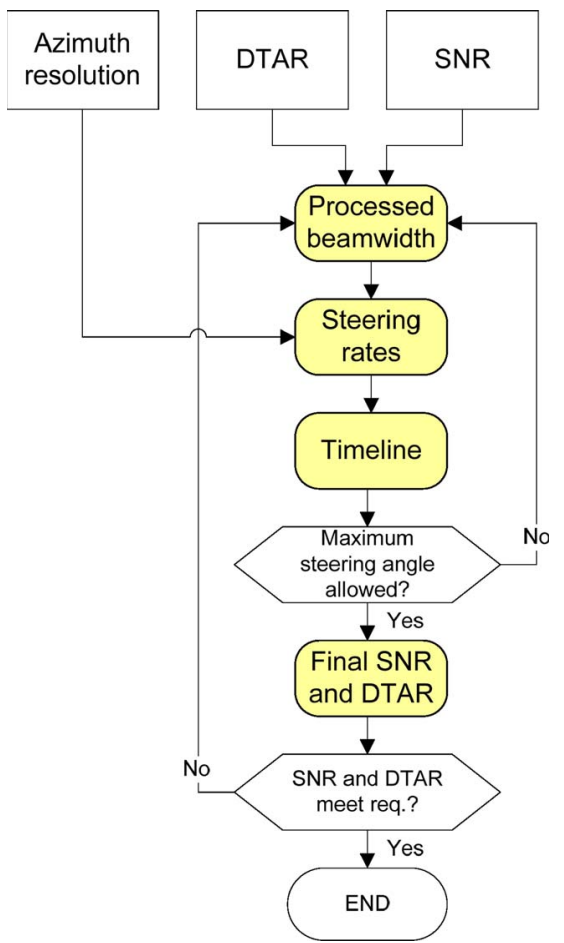

Fig. 5. Block diagram of the proposed flow to compute the parameters of the TOPS acquisition mode.

TABLE I

TSX PARAMETERS

\begin{tabular}{cc}
\hline \hline Carrier frequency & $9.65 \mathrm{GHz}$ \\
Along-track antenna length & $4.8 \mathrm{~m}$ \\
TR modules along-track & 12 \\
Azimuth beam width & $0.33^{\circ}$ \\
Orbit altitude & $514 \mathrm{~km}$ \\
Satellite velocity & $7608 \mathrm{~m} / \mathrm{s}$ \\
Maximum steering capability & $\pm 0.75^{\circ}$ \\
Number of subswaths & 4 \\
\hline \hline
\end{tabular}

Table I. Assuming a processed beamwidth of $0.33^{\circ}$, a preliminary plot for the required maximum steering as a function of the azimuth resolution and the number of subswaths is reported in Fig. 6(a), whereas Fig. 6(b) shows the required maximum steering angle rate as a function of the azimuth resolution.

An example of TOPS acquisition with TSX is reported in the following, having selected four subswaths with a total swath coverage of $100 \mathrm{~km}$, as this is the case in the TSX-ScanSAR operational mode. In this example, the value of $\vartheta_{o}^{n}$ is set equal to the 3-dB azimuth antenna beamwidth. The input and the derived parameters are listed in Table II. The intermediate results of the TOPS analysis are the variation of the DTAR and the SNR as a function of the integrated beamwidth, which are plotted in Fig. 7(a) and (b). An analysis of the TOPS DTAR variation depending on the pointing angle is performed for the TSX example. The results are shown in Fig. 7(c) and (d). A DTAR variation of less than half a decibel is expected within a burst image (the maximum steering angle is about $0.5^{\circ}$ ).

The angle quantization in TSX has been designed for azimuth steering in spotlight mode. Since, in the presented TOPS acquisition example, four subswaths have to be supported, the available 125 possible steering angles need to be distributed between the subswaths. Under this constraint, the resulting 


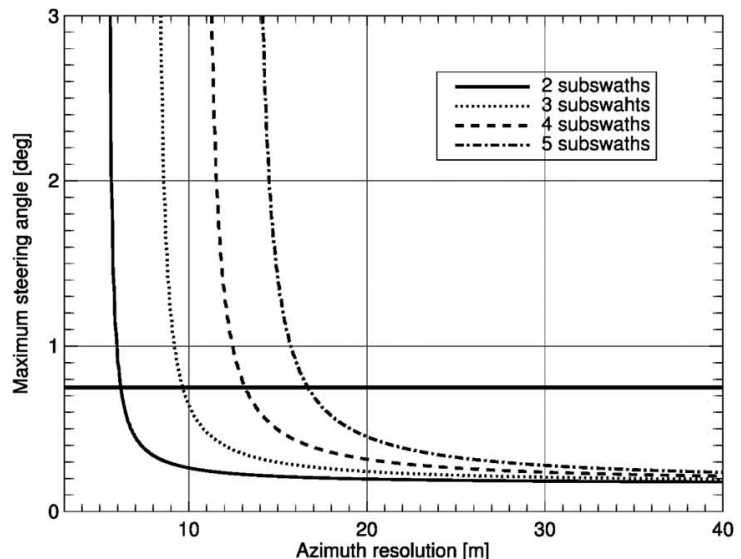

(a)

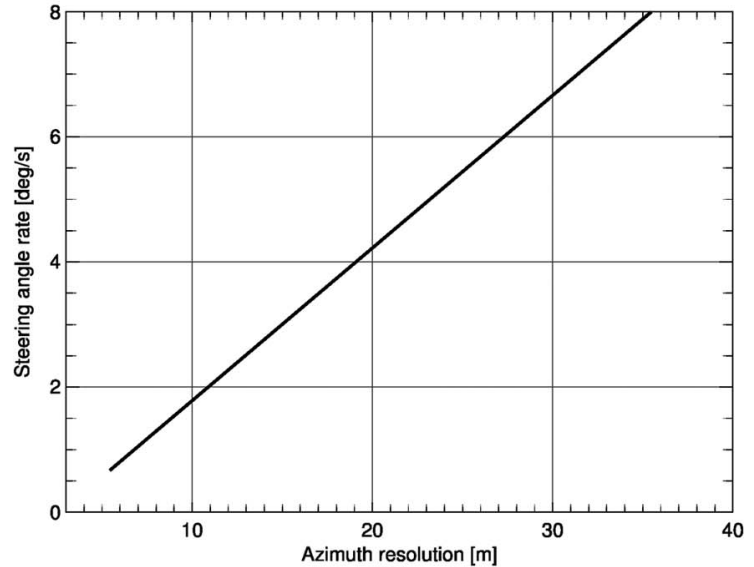

(b)

Fig. 6. (a) Maximum steering angle plotted as a function of the azimuth resolution using the TSX parameters. The different curves correspond to different number of total subswaths. The maximum TSX steering angle is also plotted (thick solid horizontal black line). (b) Maximum steering angle rate as a function of the azimuth resolution for the four subswath configurations of TSX.

angle quantization is calculated to be approximately $0.03^{\circ}$. In Fig. 8(a), the finest possible azimuth quantization in TSX is plotted as a function of the azimuth resolution and for different number of subswaths. Another constraint on the quantization angle is imposed by the dwell time. In fact, in order to guarantee a uniform SNR and DTAR, every target has to be illuminated by the whole processed antenna beam. For a certain allowed maximum SNR and DTAR variation, the azimuth antenna steering angle has to change more than a certain number of times during the dwell time. Fig. 8(b) shows the coarsest allowed angle quantization as a function of the azimuth resolution for a different number of azimuth beams (or angular steps) per target. Note that the curves do not depend on range.

Concluding, the analysis shows that TOPS can achieve the same resolution and swath coverage as ScanSAR with TSX (16m and four subswaths, respectively) with the additional benefit of a nearly constant DTAR and SNR and almost no scalloping (see Section IV-B).

\section{TOPS INTERFEROMETRY}

The TOPS mode is claimed of being useful for the generation of stacks for differential interferometric evaluations with weekly revisit time [4].
TABLE II

INPUT AND CALCULATED PARAMETERS FOR THE TOPS ACQUISITION EXAMPLE WITH TSX

\begin{tabular}{lcccc}
\hline \hline & SS 1 & SS 2 & SS 3 & SS 4 \\
\hline Input parameters & & & & \\
\hline TerraSAR-X elevation beam & 7 & 8 & 9 & 10 \\
Mean look angle [deg] & 28.4 & 30.4 & 32.0 & 34.0 \\
Slant middle range [km] & 591.8 & 604.6 & 616.5 & 632.5 \\
Ground velocity [m/s] & 6976 & 6970 & 6966 & 6960 \\
Effective radar velocity [m/s] & 7285 & 7282 & 7280 & 7276 \\
PRF [Hz] & 4063 & 3761 & 4368 & 4035 \\
Azimuth resolution [m] & 16 & 16 & 16 & 16 \\
DTAR & $-25 \mathrm{~dB}$ & $-25 \mathrm{~dB}$ & $-25 \mathrm{~dB}$ & $-25 \mathrm{~dB}$ \\
& & & & \\
\hline Calculated parameters & & & & \\
\hline Integration beam width [deg] & 0.329 & 0.329 & 0.329 & 0.329 \\
Steering angle rate [deg/s] & 3.47 & 3.40 & 3.33 & 3.25 \\
DTAR [dB] & -25.03 & -23.46 & -25.31 & -24.99 \\
Burst Time [s] & 0.300 & 0.301 & 0.303 & 0.305 \\
Dwell Time [s] & 0.078 & 0.080 & 0.082 & 0.084 \\
Target Doppler bandwidth [Hz] & 455 & 455 & 455 & 454 \\
Maximum steering angle [deg] & 0.522 & 0.513 & 0.506 & 0.497 \\
Number of pulses per burst [] & 1220 & 1136 & 1326 & 1234 \\
Burst image length [m] & 9471 & 9469 & 9467 & 9464 \\
One side burst overlap [\%] & 10.7 & 10.8 & 10.8 & 10.9 \\
\hline \hline
\end{tabular}

\section{A. Burst Alignment}

The performance that is achievable by an interferometric TOPS pair depends upon the capability to observe the same area with the same squint angle. A squint error $\delta \vartheta$ translates into a Doppler shift of

$$
f_{\text {shift }}=\frac{2 v_{s}}{\lambda} \sin (\delta \vartheta) .
$$

The total squint error is due to the contribution of both the antenna steering angle accuracy and the along-track position accuracy in the along-track timing of the individual bursts. To account for the small steering angle deviations in TOPS, there is the possibility of a tradeoff between accepting a deterioration of the SNR and the DTAR and the reduction in azimuth resolution. Note that, in ScanSAR interferometry, there will always be a reduction in resolution, as the shorter time duration of the bursts is limiting the available bandwidth. The limited alongtrack timing accuracy will lead to a reduction in resolution, as in the ScanSAR case.

In the case of TSX, the along-track pointing accuracy is usually better than $0.02^{\circ}$ (three sigma), whereas the a priori along-track position accuracy is better than $50 \mathrm{~m}$ [5] in a repeat-pass configuration, which corresponds to a squint angle error of $0.005^{\circ}$. Therefore, the worst case total squint angle error is assumed as $0.025^{\circ}$, leading to a Doppler shift of approximately $210 \mathrm{~Hz}$. For the TSX TOPS configuration (16-m azimuth resolution), the focused Doppler bandwidth $B_{D}$ is $455 \mathrm{~Hz}$; therefore, the worst case interferometric resolution loss is $46 \%$. If the pointing inaccuracy is traded off against the degraded SNR and the DTAR by processing one of the acquisitions with the same mean Doppler centroid as the other, the maximum relative interferometric resolution loss is determined by the along-track timing only and is $9 \%$.

\section{B. Coregistration Accuracy}

As in ScanSAR interferometry, the special TOPS signal properties must be understood and accounted for in order to 


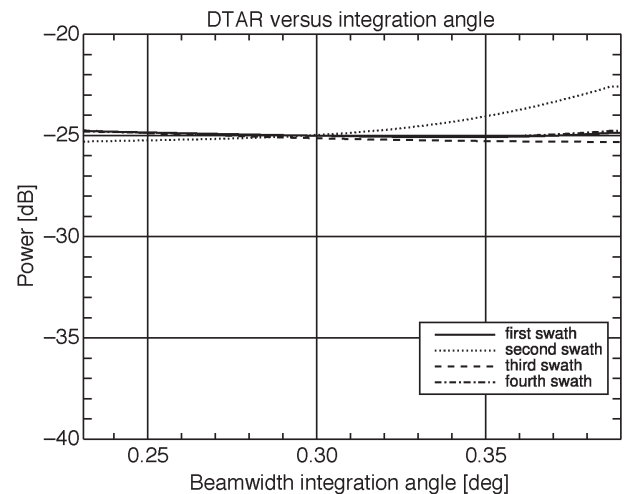

(a)

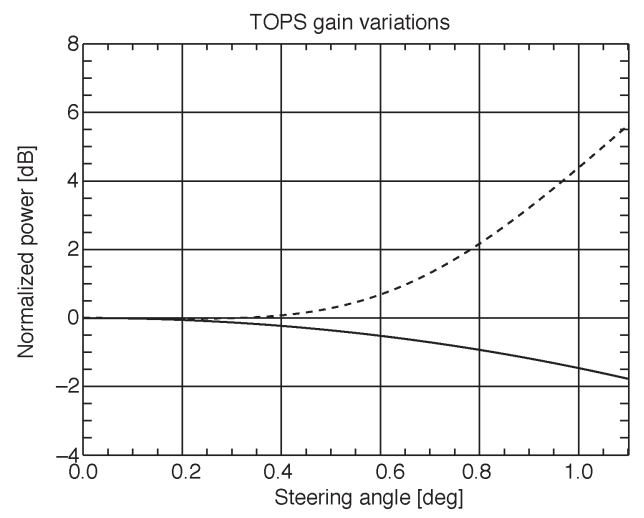

(c)

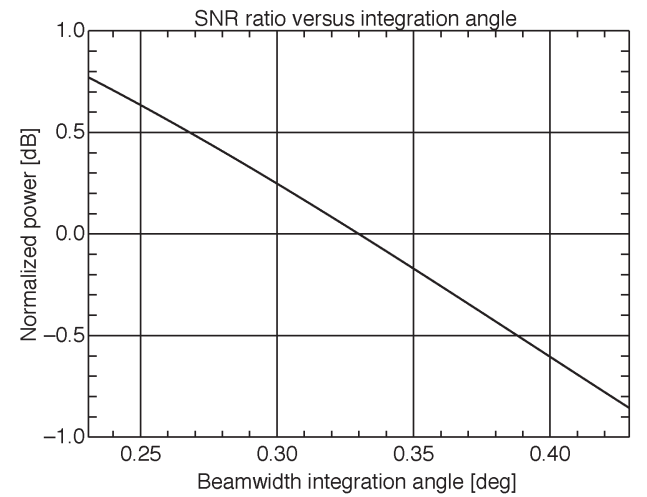

(b)

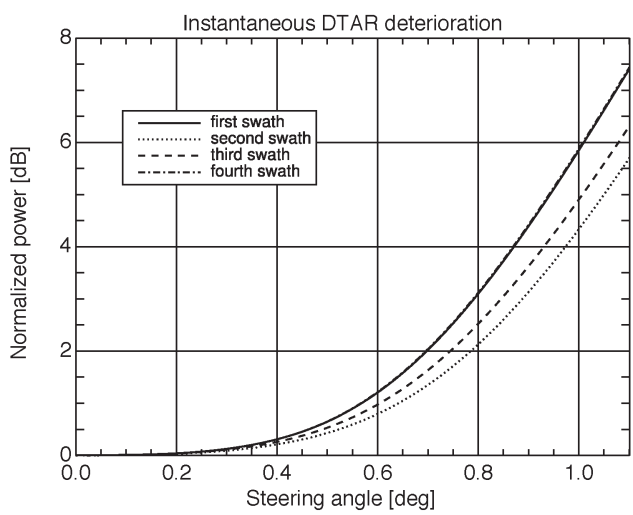

(d)

Fig. 7. (a) TSX DTAR values calculated as a function of the beamwidth integration angle for a boresight case. (b) TSX SNR variation calculated as a function of the beamwidth integration angles for a boresight case. The SNR values are normalized with respect to the SNR obtained, integrating the one-way 3-dB beamwidth. (c) (Solid line) TOPS main lobe and (dashed line) azimuth ambiguity power variation depending on the steering angle for the first subswath of the TSX configuration example. The power variation is normalized to the boresight case. (d) TOPS DTAR deterioration depending on the steering angle for the subswaths of the TSX configuration example. The power loss is normalized to the boresight case.

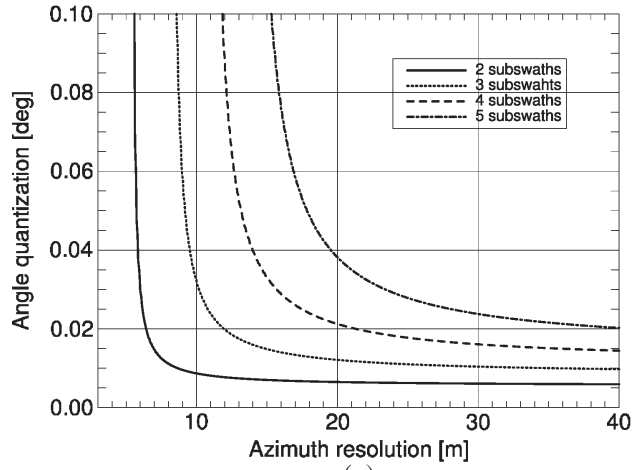

(a)

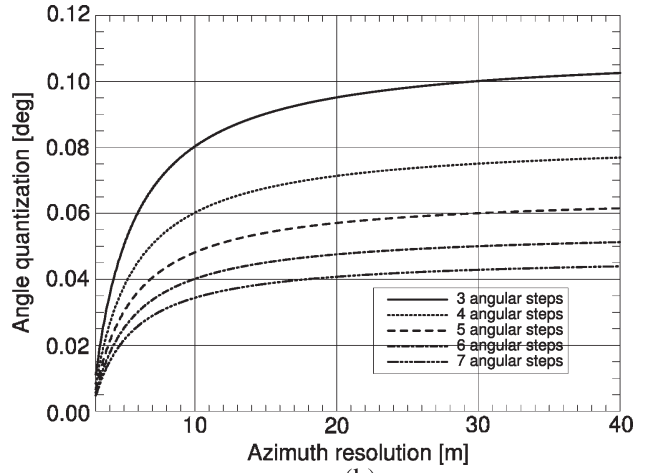

(b)

Fig. 8. (a) Minimum angle quantization satisfying the constraint imposed by the TSX limited number of steering angles per data take (125 angles) plotted as a function of the azimuth resolution. (b) Maximum-allowed angle quantization as a function of the azimuth resolution and for different values of angular steps per target. The number of azimuth steps results from the allowed variation of SNR and DTAR. The curves do not depend on range.

obtain reliable interferometric products [7], [8], [11]. One of the characteristics of the TOPS acquisition mode is that the data are acquired with high Doppler centroid variations within a burst. For typical TSX TOPS acquisitions, the Doppler centroid can vary by more than $10 \mathrm{kHz}$ within one burst. It is well known that, in the presence of a squint, linear phase ramps are induced in the focused response both in azimuth and range. Thus, constant misregistration can cause the presence of alongtrack and across-track linear phase ramps [8], [9].

Range Coregistration Accuracy: The expression for the interferometric phase error induced by a linear ramp of the impulse response in the range direction when range misregistration is present is [9]

$$
\varphi_{\mathrm{rg} \_ \text {err }}=\frac{4 \pi}{\lambda} \Delta r_{\text {mis }} \cdot\left(1-\sqrt{1-\left(\frac{\lambda f_{\mathrm{DC}}\left(R_{0}\right)}{2 v}\right)^{2}}\right) .
$$

For the TSX parameters, this expression results in negligible values that are smaller than $0.1 \mathrm{rad}$; therefore, TOPS does not introduce any additional stringent requirement for the range coregistration accuracy in interferometry applications. 
Azimuth Coregistration Accuracy: Since each TOPS burst is acquired with a varying Doppler centroid, every focused point presents a different linear phase ramp in the azimuth direction. The slope of the ramp depends on the Doppler centroid. The resulting interferometric TOPS phase bias in the presence of azimuth misregistration is similar to the ScanSAR bias, and it is equal to [8]

$$
\varphi_{\mathrm{az} \_ \text {err }}=2 \pi f_{\mathrm{DC}} \Delta t .
$$

Within a burst, this corresponds to a linear phase term along azimuth since $f_{\mathrm{DC}}$ is a function of the azimuth position within the burst. For a TSX acquisition with a Doppler variation of $10.35 \mathrm{kHz}$, and a pixel spacing of $8.69 \mathrm{~m}$, a misregistration of 0.1 pixel introduces a ramp of approximately $2.5 \pi$ within the burst. Therefore, an overall azimuth coregistration accuracy of better than 0.001 of the pixel is required for this configuration in order to achieve an error that is smaller than $10^{\circ}$. Note, however, that this required accuracy applies mainly to a constant coregistration offset for the whole burst, and the achievable relative coregistration accuracy can be much better than this requirement. First, a coregistration can be performed either using the orbit's information and an external digital elevation model, amplitude cross correlation, or coherence maximization, all of which yield accuracies that are better than one pixel. Then, a fine coregistration using spectral diversity [8] would result in the required fine accuracy. When necessary, very fine coregistration accuracy can be obtained by using again spectral diversity in the overlapping region of two consecutive TOPS bursts. In this case, the sensitivity to the remaining error is much larger due to the larger separation between spectral looks.

\section{EXPERIMENTAL RESULTS}

This section presents first a TSX image processing example, including all four subswaths. Next, a quantitative analysis of the scalloping for TOPS, inverse TOPS, and ScanSAR acquisition modes based on the TSX data acquired in these three different imaging modes is presented. Finally, the feasibility of TOPS interferometry is demonstrated.

\section{A. TOPS Image Result With TSX}

Fig. 9 shows a TOPS data take over Barcelona, Spain. The absence of scalloping is evident. It should be noted that no antenna pattern correction was applied to remove the residual scalloping due to the electronic steering. The data were processed using the baseband azimuth scaling approach [6], [20]. This image was used to analyze the response of the calibration targets to confirm the expected performance in terms of resolution, peak sidelobe ratio, and point target ambiguity ratio. Furthermore, no indication of scalloping was found in the image, not even in the open sea.

\section{B. Results of the Scalloping Image Analysis}

Three TSX images have been acquired over Toulouse, France, in order to perform a scalloping analysis in the TOPS, inverse TOPS, and ScanSAR modes [12]. Fig. 10 presents the first subswath out of the four from the three modes processed without any scalloping correction. The azimuth resolution in

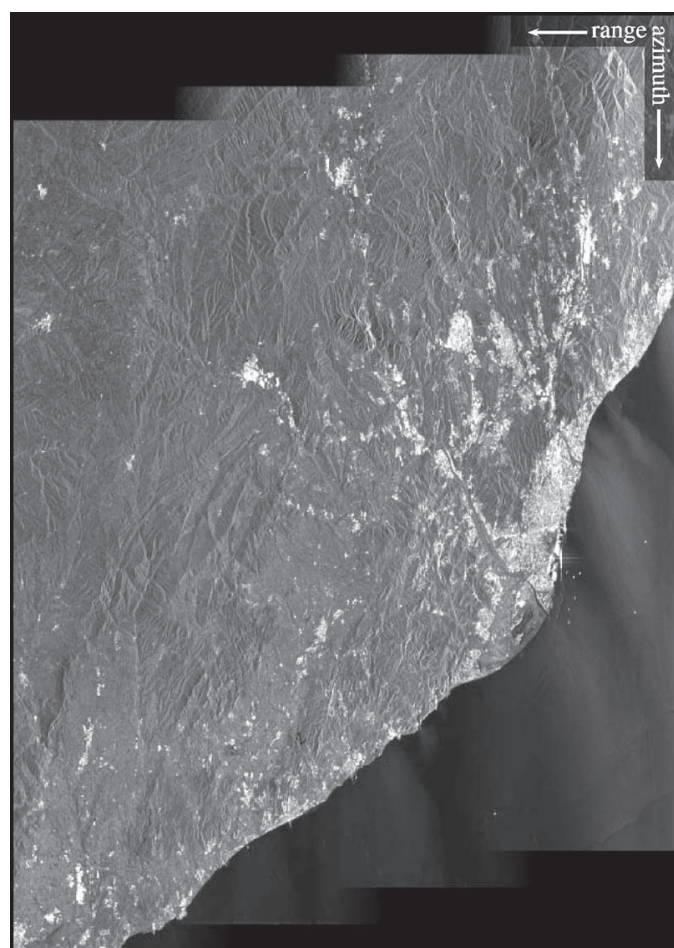

Fig. 9. TOPS data take over Barcelona, Spain, acquired on December 28, 2007, by TSX.

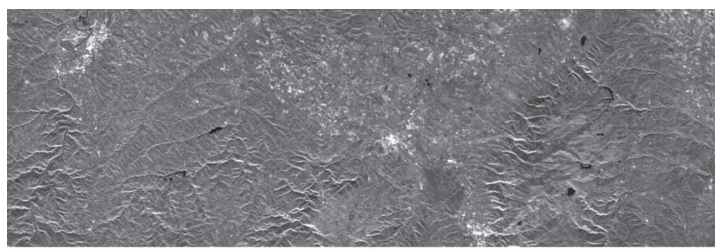

(a)

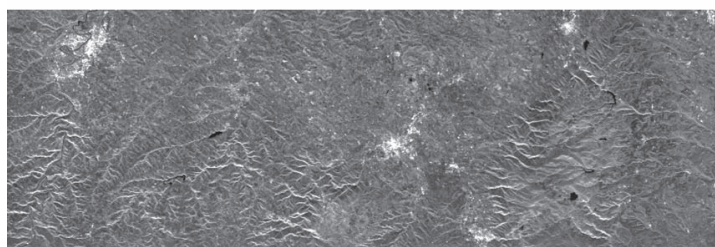

(b)

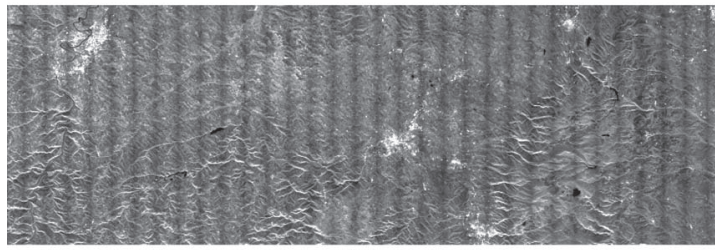

(c)

Fig. 10. (a) TOPS, (b) inverse TOPS, and (c) ScanSAR comparison. The first subswath is shown without scalloping correction. The measured scalloping in the ScanSAR image is around $1.2 \mathrm{~dB}$ compared to $0.7 \mathrm{~dB}$ in inverse TOPS and $0.3 \mathrm{~dB}$ in the TOPS image. Thirty-three ScanSAR bursts were necessary in contrast to only nine required by the two TOPS modes.

the images is $16 \mathrm{~m}$. About five range looks were processed, resulting in a 16-m ground range resolution. The subswath size is approximately $90 \mathrm{~km}$ in azimuth and $25 \mathrm{~km}$ in slant range. No weighting has been applied in the burst image overlap area.

The TOPS image subswath shown in Fig. 10(a) was acquired with nine bursts. The commanded steering angle is in-between 


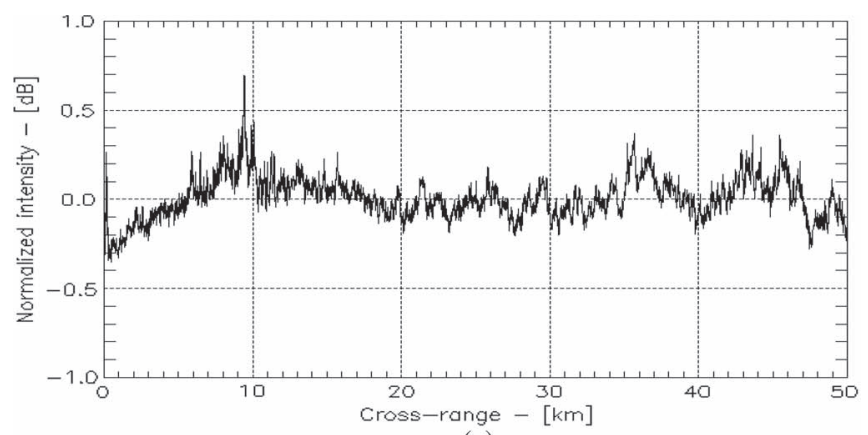

(a)

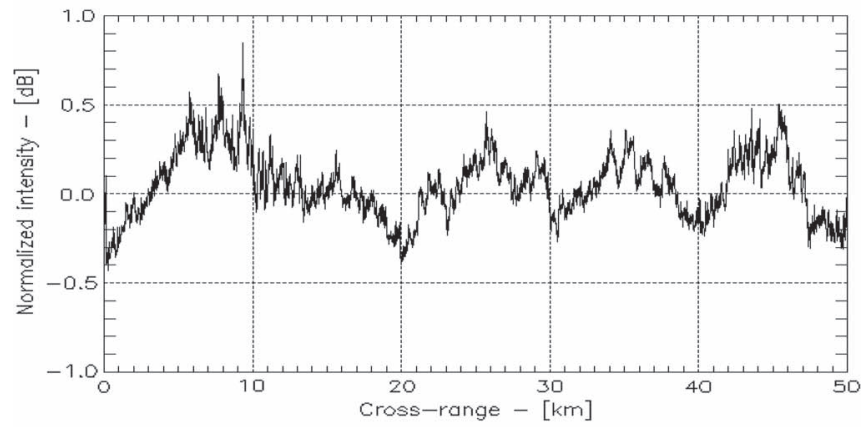

(b)

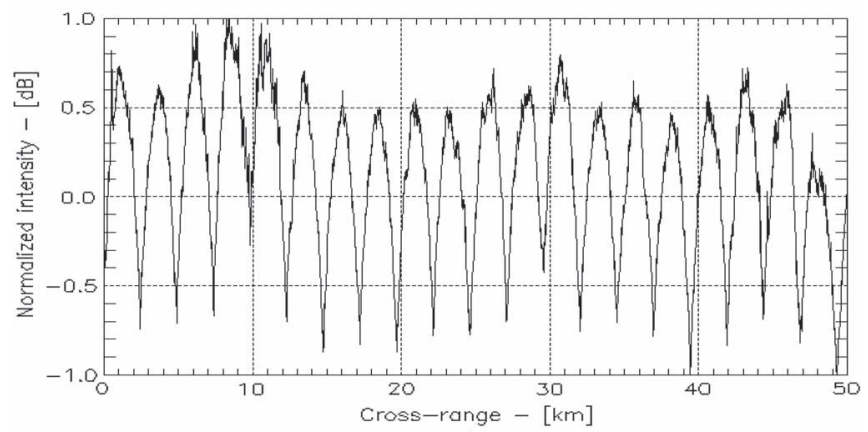

(c)

Fig. 11. Intensity azimuth profiles extracted from part of the first subswath of the (a) TOPS, (b) inverse TOPS, and (c) ScanSAR image. No azimuth pattern correction was applied in the processing.

$\pm 0.52^{\circ}$. Due to the low variation of the steering angle, the scalloping effect is hardly visible, and it was quantified to be approximately $0.3 \mathrm{~dB}$ from the azimuth profile in Fig. 11(a).

In inverse TOPS, the commanded steering angle is inbetween $\pm 0.73^{\circ}$. A small scalloping effect is visible in the inverse-TOPS image. A variation of approximately $0.7 \mathrm{~dB}$ has been measured from the generated azimuth profile in Fig. 11(b). Therefore, as indicated in Section II-B, the TOPS mode is always preferable to the inverse TOPS mode in systems with electronically steered array antennas.

The ScanSAR image was acquired with 33 bursts. The measured scalloping in the ScanSAR intensity profile in Fig. 11(c) is around $1.2 \mathrm{~dB}$.

The advantage in using the TOPS technique in terms of scalloping and, therefore, SNR is clearly visible.

\section{First Interferometric Results}

This section presents the first TOPS interferometric results [13]. Two TOPS data takes have been acquired over a flat and high coherent region. The chosen area is Uyuni salt lake, Bolivia, one of the largest in the world. The data takes were

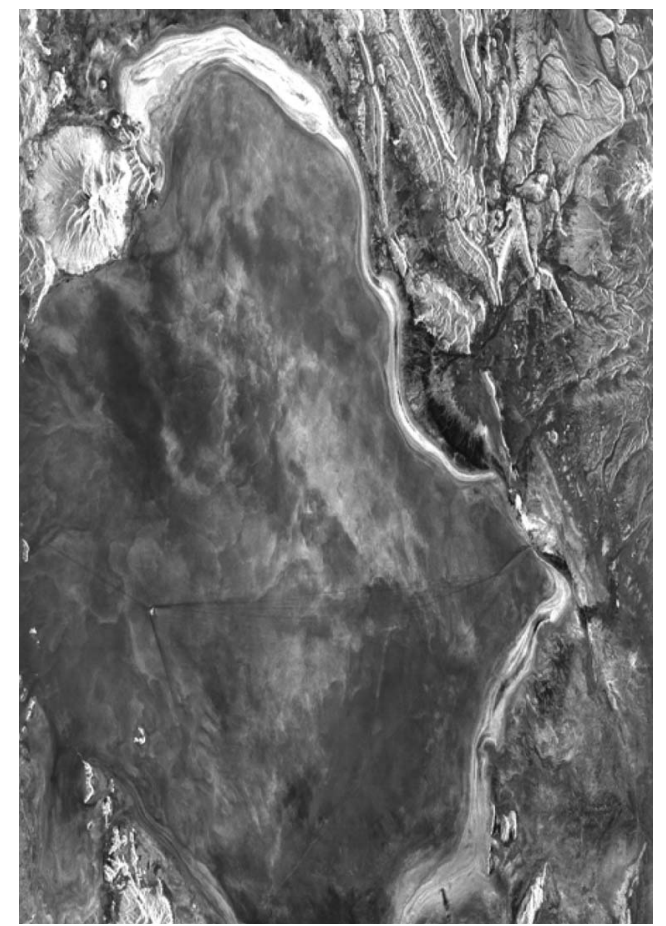

Fig. 12. TOPS amplitude image used as master for the interferometric analysis. The data take has been acquired by TSX on October 10, 2007, over Uyuni salt lake, Bolivia.

recorded on October 10 and 21, 2007, with perpendicular baselines varying from 67 to $38 \mathrm{~m}$. The master TOPS image is shown in Fig. 12. The data have been acquired with four subswaths and eight bursts. The look angle is varying from $32.9^{\circ}$ to $37.9^{\circ}$. The slant range swath width is $74 \mathrm{~km}$, while the scene azimuth extension is $110 \mathrm{~km}$. The pixel spacing is $8.69 \mathrm{~m}$. Around the salt lake, it is possible to see mountainous areas, whereas a small island is visible within the lake.

For the TOPS interferometry analysis reported in this paper, each burst has been coregistered with an accuracy of 0.1 pixel spacing by coherence maximization techniques. The mean of the estimated along-track shift between the two acquisitions is around $20 \mathrm{~m}$, resulting in a resolution loss of only $4 \%$. By using this information, the slave TOPS image has been reprocessed in order to maximize the Doppler spectrum overlap and reduce azimuth fringes induced by the azimuth misregistration. The resulting coherence picture is shown in Fig. 13, with a maximum coherence value of 0.94 in the high-reflectivity salt lake areas. The peak of the coherence histogram is at 0.79 . A small degradation of coherence is noticeable at each range subswath edge due to the variation of the antenna elevation gain. The same effect would be present also in ScanSAR coherence images. Finally, Fig. 14 shows the final flattened interferogram.

\section{CONCLUSION}

This paper has reported the system design aspects for the TOPS mode with an electronically steered antenna. A comparison to the ScanSAR mode of operation has been made, and the inverse TOPS mode has been discussed. The effects to be considered in SAR systems with an electronically steered antenna have been introduced and discussed, i.e., steering angle quantization, presence of grating lobes, and antenna main lobe 


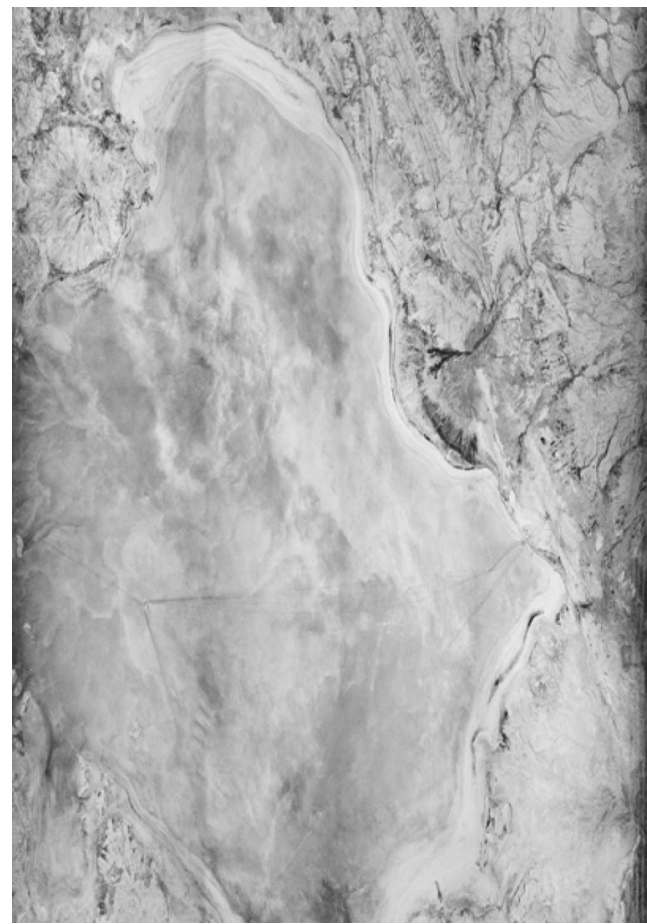

Fig. 13. TOPS interferometric coherence image over Uyuni salt lake, Bolivia. Coherence values between (black) zero and (white) one.

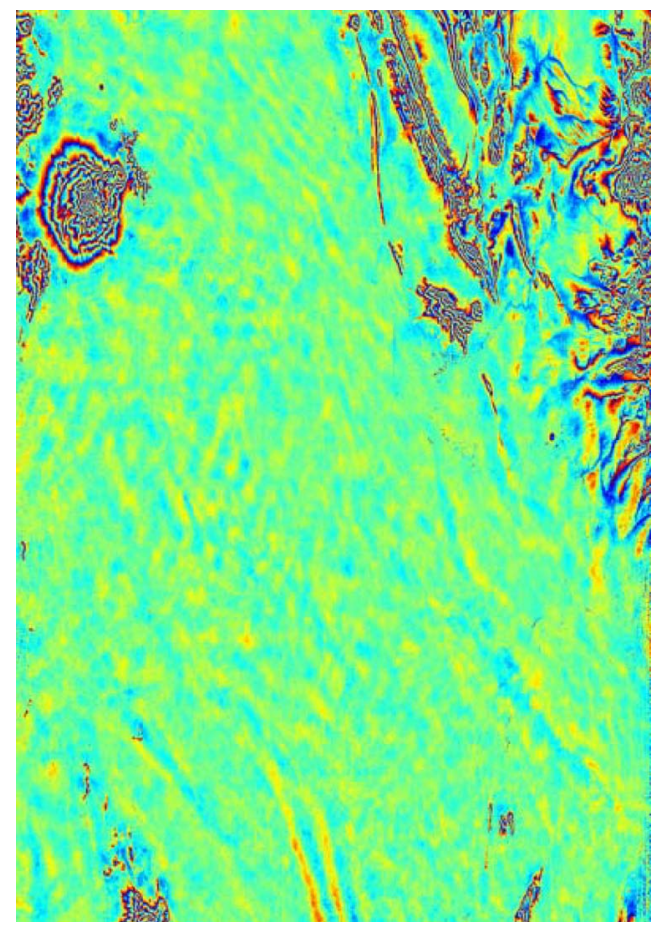

Fig. 14. TOPS interferometric phase image over Uyuni salt lake, Bolivia.

attenuation, leading to an azimuth variation of the SNR and the azimuth ambiguity ratio, as well as scalloping.

This paper has presented the system design parameter calculation flow and the resulting parameter values for a TSX acquisition example. The special characteristics of TOPS interferometry have been discussed, and the requirements to the coregistration accuracy have been formulated.
A quantitative comparison of TOPS, inverse TOPS, and ScanSAR images has been performed in terms of scalloping. The measured values of the intensity variation of the analyzed images correspond very well with the expected theoretical values. Scalloping in the TOPS image is $0.3 \mathrm{~dB}$ against $1.2 \mathrm{~dB}$ in the ScanSAR image. Additionally, fewer bursts are required in TOPS, which also positively affects the image quality.

The TSX steering capability allows TOPS operations with the same coverage and resolution as the nominal ScanSAR but without the ScanSAR scalloping and the azimuth-dependent performance effects. The most important design parameter in the development of new instruments with TOPS mode is the maximum required steering angle in azimuth which is driven by azimuth resolution and the gap-free subswath acquisition.

Several data takes with TSX TOPS and inverse TOPS have been acquired and processed with the experimental TOPS processor developed at the German Aerospace Center [6], [20], and first interferometric results have been presented in this paper.

\section{APPENDIX}

This appendix shows the derivation of the analytical error introduced by a quantization of the steering law. The amplitude error for a given target is given by the difference between the ideal antenna pattern and the quantized one. This error can be modeled as a saw tooth signal, defined as

$$
x_{\mathrm{st}}(t)=A_{e} \sum_{n} \operatorname{rect}\left(\frac{t-n T_{q}}{T_{q}}\right) \cdot\left(t-n T_{q}\right)
$$

where $A_{e}$ is the worst case amplitude error and $T_{q}$ is the period of the error signal that is equal to

$$
T_{q}=\frac{\Delta \vartheta}{k_{\vartheta}}
$$

with $k_{\vartheta}$ being the steering rate and $\Delta \vartheta$ being the quantization step size. The advantage of using this model lies on the fact that it is easy to derive an analytical expression of its Fourier transform, whose amplitude is equal to

$$
\left|X_{\mathrm{st}}(f)\right|=\frac{A_{e}}{\pi\left|f T_{q}\right|} \int \sum_{n \neq 0} \delta\left(f-\frac{n}{T_{q}}\right) \partial f
$$

which directly yields the IRF of the target. In a practical situation, the delta function is replaced by the sinc function due to the finite observation time. The term $A_{e}$ represents the maximum value of the error amplitude; this value approximately corresponds to the difference between the uniform and the quantized antenna gain calculated at the $3-\mathrm{dB}$ point

$$
A_{e}=\operatorname{sinc}^{2}\left(\frac{1}{2}\right)-\operatorname{sinc}^{2}\left(\frac{1}{2}-\frac{\Delta \vartheta}{2 \vartheta_{3 \mathrm{~dB}}}\right) .
$$

Using (15) in (14), it is possible to retrieve the amplitude of the first spurious response as a function of the angle quantization, which is then given by

$$
\begin{aligned}
S_{q}(\Delta \vartheta) & =\max \left|X_{\mathrm{st}}(f)\right|_{\Delta \vartheta} \\
& =\frac{A_{e}}{\pi}=\frac{\operatorname{sinc}^{2}\left(\frac{1}{2}\right)-\operatorname{sinc}^{2}\left(\frac{1}{2}-\frac{\Delta \vartheta}{2 \vartheta_{3 \mathrm{~dB}}}\right)}{\pi} .
\end{aligned}
$$




\section{ACKNOWLEDGMENT}

The authors would like to thank Evert Attema and Paul Snoeij of the European Space Research and Technology Centre, ESA, for the useful discussions in the frame of the TOPS project and Steffen Wollstadt for the fruitful discussions with respect to the commanding of TOPS with TSX.

\section{REFERENCES}

[1] F. De Zan and A. Monti Guarnieri, "TOPS: Terrain observation by progressive scan," IEEE Trans. Geosci. Remote Sens., vol. 44, no. 9, pp. 2352-2360, Sep. 2006.

[2] E. Attema, M. Davidson, N. Floury, G. Levrini, B. Rosich, B. Rommen, and P. Snoeij, "Sentinel-1 ESA's new European radar observatory," in Proc. EUSAR, Friedrichshafen, Germany, Jun. 2-5, 2008, vol. 2, pp. 179-182.

[3] Sentinel-1 TOPS Imaging Mode Demonstration With TerraSAR-X, ESA contract C20679/07/NL/BC

[4] F. De Zan, D. d'Aria, A. Monti Guarnieri, and F. Rocca, "Spaceborne DINSAR monitoring with weekly revisit time with TOPS," in Proc. EUSAR, Dresden, Germany, 2006.

[5] J. Mittermayer, D. Schulze, U. Steinbrecher, and J. Marquez-Martinez, "The system engineering and calibration segment of the TerraSAR-X ground segment," in Proc. IEEE IGARSS, Seoul, Korea, Jul. 2005, pp. $4878-4881$.

[6] P. Prats, R. Scheiber, J. Mittermayer, A. Meta, J. Sanz-Marcos, and A. Moreira, "A SAR processing algorithm for TOPS imaging mode based on extended chirp scaling," in Proc. IEEE IGARSS, Barcelona, Spain, Jul. 23-27, 2007, pp. 148-151.

[7] A. Monti Guarnieri and C. Prati, "ScanSAR focusing and interferometry," IEEE Trans. Geosci. Remote Sens., vol. 34, no. 4, pp. 1029-1038, Jul. 1996.

[8] R. Scheiber and A. Moreira, "Coregistration of interferometric SAR images using spectral diversity," IEEE Trans. Geosci. Remote Sens., vol. 38, no. 5, pp. 2179-2191, Jul. 2000.

[9] M. Bara, R. Scheiber, A. Broquetas, and A. Moreira, "Interferometric SAR signal analysis in the presence of squint," IEEE Trans. Geosci. Remote Sens., vol. 38, no. 5, pp. 2164-2178, Sep. 2000.

[10] A. Moreira, J. Mittermayer, and R. Scheiber, "Extended chirp scaling algorithm for air- and spaceborne SAR data processing in stripmap and ScanSAR imaging modes," IEEE Trans. Geosci. Remote Sens., vol. 34, no. 5, pp. 1123-1136, Sep. 1996.

[11] J. Holzner and R. Bamler, "Burst-mode and ScanSAR interferometry," IEEE Trans. Geosci. Remote Sens., vol. 40, no. 9, pp. 1917-1934, Sep. 2002.

[12] A. Meta, P. Prats, U. Steinbrecher, J. Mittermayer, and R. Scheiber, "TerraSAR-X TOPS and ScanSAR comparison," in Proc. EUSAR, Friedrichshafen, Germany, 2008.

[13] A. Meta, P. Prats, U. Steinbrecher, R. Scheiber, and J. Mittermayer, "First TOPS interferometry results with TerraSAR-X," in Proc. FRINGE, Frascati, Italy, 2007.

[14] A. Meta, J. Mittermayer, U. Steinbrecher, and P. Prats, "Investigations on the TOPS acquisition mode with TerraSAR-X," in Proc. IEEE IGARSS, Barcelona, Spain, 2007, pp. 152-155.

[15] D. D'Aria and D. Giudici, "Tops-SAR system analysis and optimization," GMES Sentinel-1 project, ESA-ESTEC Presentation, The Netherlands, Mar. 2006.

[16] D. D'Aria, F. De Zan, D. Giudici, A. Monti Guarnieri, and F. Rocca, "Burst-mode SARs for wide-swath surveys," Can. J. Remote Sens., vol. 33, no. 1, pp. 27-38, Feb. 2007.

[17] R. Werninghaus and S. Buckkreuß, "The TerraSAR-X mission and system design," IEEE Trans. Geosci. Remote Sens., vol. 48, no. 2, pp. 606-614, Feb. 2010

[18] S. Buckreuss and B. Schättler, "The TerraSAR-X ground segment," IEEE Trans. Geosci. Remote Sens., vol. 48, no. 2, pp. 623-632, Feb. 2010.

[19] U. Steinbrecher, D. Schulze, J. Böer, and J. Mittermayer, "TerraSAR-X instrument operations rooted in the system engineering and calibration project," IEEE Trans. Geosci. Remote Sens., vol. 48, no. 2, pp. 633-641, Feb. 2010

[20] P. Prats, R. Scheiber, J. Mittermayer, A. Meta, and A. Moreira, "Processing of sliding spotlight and TOPS SAR data using baseband azimuth scaling," IEEE Trans. Geosci. Remote Sens., vol. 48, no. 2, pp. 770-780, Feb. 2010.

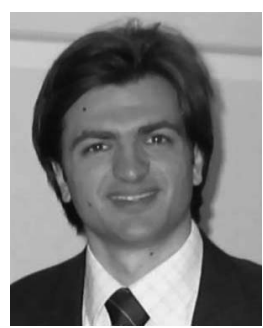

Adriano Meta (S'01-M'05) was born in Pontecorvo, Italy, in 1978. He received the Laurea degree in telecommunication engineering from the Sapienza, Universitá di Roma, Rome, Italy, in 2002, with the maximum grade and within the best $0.5 \%$ of the students, and the Ph.D. degree (cum laude) from the Delft University of Technology, Delft, The Netherlands, in 2006.

In 2000, he spent an academic year with Ghent University of Technology, Ghent, Belgium. From 2002 to 2006, he was with the International Research Centre for Telecommunications and Radar, Delft University of Technology. $\mathrm{He}$ has attended the post lauream Aerospace School, Sapienza, Universitá di Roma. From 2006 to 2008, he was with the Microwaves and Radar Institute, German Aerospace Center, Oberpfaffenhofen, Germany, where he was involved in the commissioning phase of TerraSAR-X (TSX) and in the development of advanced processing techniques for digital beamforming and multichannel radar applications. He was the Deputy Manager of the demonstration project of the Terrain Observation by Progressive Scan (TOPS) acquisition mode with TSX. He is currently the Director of MetaSensing, Noordwijk, The Netherlands, a Dutch company that he founded in 2008 to develop and commercialize compact high-resolution low-cost airborne synthetic aperture radar (SAR) sensors and services. MetaSensing is supported by the European Space Agency through the Business Incubation program. He is the author of several papers on frequency-modulated continuous-wave (FMCW) SAR signal processing, advanced SAR techniques, and hardware design. He is the holder of an international patent on FMCW SAR frequency nonlinearity correction.

Dr. Meta was the recipient of the Student Paper prizes at Waveform Diversity and Design Conference'06, European Synthetic Aperture Radar Conference'06, International Geoscience and Remote Sensing Symposium'06, and European Radar Conference'06.

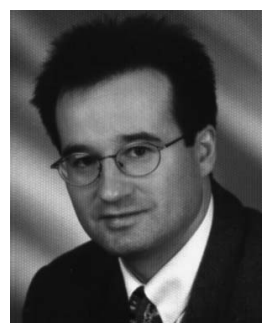

Josef Mittermayer (M'07) was born in Wartenberg, Oberbayern, Germany, in 1967. He received the Dipl.-Ing. (M.Sc.) degree in electrical engineering from the Technical University of Munich, Germany, in 1995, with a thesis on ScanSAR processing, the Dr.-Ing. (Ph.D.) degree in spotlight synthetic aperture radar (SAR) processing from the University of Siegen, Germany, in 2000, and the M.Sc. degree in space system engineering from the Delft University of Technology, Delft, The Netherlands, in 2004.

From 1994 to 2001, he was with the Signal Processing Group, Microwaves and Radar Institute, German Aerospace Center (DLR), Oberpfaffenhofen, Germany. Since 2002, he has been working in the TerraSAR Project at DLR. From January 2004 until the end of the commissioning phase in 2008, he was the Group Leader and the Project Manager of system engineering and calibration, one of the three subprojects which form the TerraSAR-X Ground Segment. In addition, he was technically responsible for the TerraSAR-X commissioning phase. Since July 2008, he has been leading the SAR System Engineering Group, Microwaves and Radar Institute, DLR.

Dr. Mittermayer and his colleagues received the Geoscience and Remote Sensing Society Transactions Prize Paper Award for a paper on air- and spaceborne stripmap and ScanSAR processing in 1996. He was the recipient of the 2001 DLR Science Award for his work on spotlight SAR processing. 


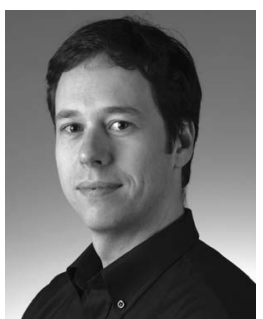

Pau Prats (S'03-M'06) was born in Madrid, Spain, in 1977. He received the Ingeniero (M.Sc.) degree in telecommunication engineering and the Ph.D. degree from the Universitat Politècnica de Catalunya (UPC), Barcelona, Spain, in 2001 and 2006, respectively.

In 2001, he was with the Institute of Geomatics, Spain, as a Research Assistant designing a subaperture synthetic aperture radar (SAR) processor. In 2002, he was with the Department of Signal Theory and Communications, UPC, where he worked in the field of airborne repeat-pass interferometry and airborne differential SAR interferometry. From December 2002 to August 2006, he was an Assistant Professor with the Department of Telecommunications and Systems Engineering, Universitat Autònoma de Barcelona, Barcelona. In 2006, he joined the Microwaves and Radar Institute, German Aerospace Center (DLR), Oberpfaffenhofen, Germany, where since August 2009 he is head of the Multimodal Algorithms Group. His research interests include air- and spaceborne SAR processing, SAR interferometry, differential SAR interferometry and motion compensation for airborne systems.

Dr. Prats was the recipient of the First Prize of the Student Paper Competition of the IEEE International Geoscience and Remote Sensing Symposium 2005 Conference held in Seoul, Korea.

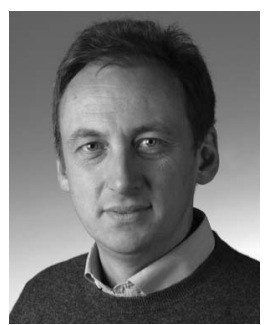

Rolf Scheiber received the Dipl.-Ing. (M.Sc.) degree in electrical engineering from the Technical University of Munich, Germany, in 1994 and the Ph.D. degree in electrical engineering from the University of Karlsruhe, Karlsruhe, Germany, in 2003, with a thesis on airborne synthetic aperture radar (SAR) interferometry.

Since 1994, he has been with the Microwaves and Radar Institute, German Aerospace Center, where he developed the operational high-precision interferometric SAR processor for its E-SAR airborne sensor. Since 2001, he has been heading the SAR Signal Processing Group, SAR Technology Department, where he is currently responsible for the E-SAR campaign data processing, as well as the development of the processing software for the new airborne sensor F-SAR. His current research interests include algorithm development for high-resolution air- and spaceborne SAR focusing, SAR interferometry, differential SAR interferometry, and SAR tomography, as well as radio sounding algorithms and applications.

Dr. Scheiber was awarded as coauthor with the 1996 Geoscience and Remote Sensing Society Transactions Prize Paper Award for the contribution "Extended Chirp Scaling Algorithm for Air- and Spaceborne SAR Data Processing in Stripmap and ScanSAR Imaging Modes" in 1997.

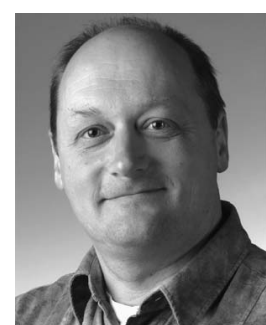

Ulrich Steinbrecher received the Dipl.Ing. (M.Sc.) degree in electrical engineering/communication from the University of Siegen, Germany, in 1990.

In 1990, he was with the German Aerospace Center (DLR), Oberpfaffenhofen, Germany, with the development of a synthetic aperture radar (SAR) raw data simulator. In 1994, he worked in the software development for the X-SAR processor for the joint U.S.-Italian-German SIR-C/X-SAR Missions. When the data were in-house, he concentrated on the aspects of operational SAR processing of high data volumes. In 1995, he pioneered a completely automatic SAR processing system based on a robot-maintained mass memory archive. Before he became responsible for the development of the raw data analysis and screening system of the Shuttle Radar Topography Mission (SRTM), he developed the software for a phase-preserving ScanSAR processor for Radarsat-1. In the time between the SRTM and the start of the TerraSAR-X (TSX) project, he left for two years the SAR domain and worked in the image processing for the atmospheric research instrument SCIAMACHY. Since 2002, he has been concerned with the TSX radar system, and, since the launch in 2007, he has been responsible for TSX Instrument Operations. He is currently with the Microwaves and Radar Institute, DLR 